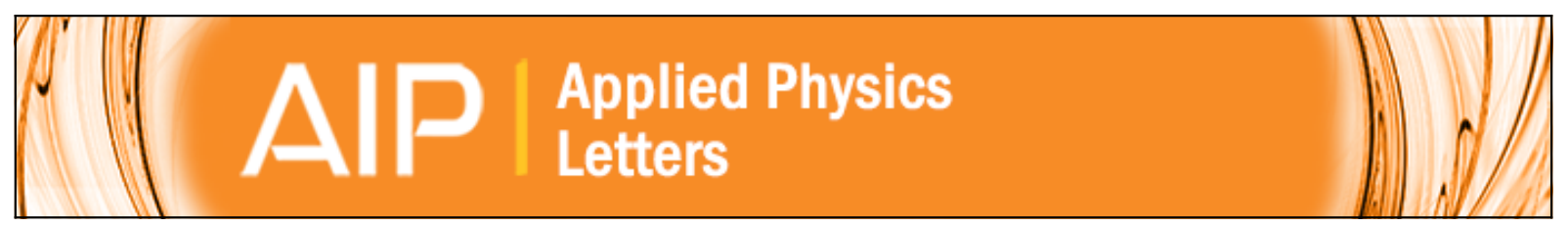

Damage and strain in single-layer graphene induced by very-low-energy electron-beam irradiation

Katsuhisa Murakami, Takuya Kadowaki, and Jun-ichi Fujita

Citation: Applied Physics Letters 102, 043111 (2013); doi: 10.1063/1.4790388

View online: http://dx.doi.org/10.1063/1.4790388

View Table of Contents: http://scitation.aip.org/content/aip/journal/apl/102/4?ver=pdfcov

Published by the AIP Publishing

$\underset{\substack{\text { Publoning } \\ \text { PIP }}}{A}$ Re-register for Table of Content Alerts

Create a profile.

Sign up today! 


\title{
Damage and strain in single-layer graphene induced by very-low-energy electron-beam irradiation
}

\author{
Katsuhisa Murakami, ${ }^{1,2, a)}$ Takuya Kadowaki, ${ }^{1}$ and Jun-ichi Fujita ${ }^{1,2}$ \\ ${ }^{1}$ Institute of Applied Physics, Graduate School of Pure and Applied Sciences, University of Tsukuba, \\ 1-1-1 Tennodai, Tsukuba, Ibaraki 305-8573, Japan \\ ${ }^{2}$ Tsukuba Research Center for Interdisciplinary Materials Science, University of Tsukuba, \\ 1-1-1 Tennodai, Tsukuba, Ibaraki 305-8573, Japan
}

(Received 27 September 2012; accepted 22 January 2013; published online 30 January 2013)

\begin{abstract}
From the analysis of the ratio of $\mathrm{D}$ peak intensity to $\mathrm{G}$ peak intensity in Raman spectroscopy, electron beam irradiation with energies of $100 \mathrm{eV}$ was found to induce damage in single-layer graphene. The damage becomes larger with decreasing electron beam energy. Internal strain in graphene induced by damage under irradiation is further evaluated based on $\mathrm{G}$ peak shifts. The dosedependent internal strain was approximately $2.22 \% \mathrm{~cm}^{2} / \mathrm{mC}$ at $100 \mathrm{eV}$ and $2.65 \times 10^{-2} \% \mathrm{~cm}^{2} / \mathrm{mC}$ at $500 \mathrm{eV}$. The strain induced by the irradiation showed strong dependence on electron energy. (C) 2013 American Institute of Physics. [http://dx.doi.org/10.1063/1.4790388]
\end{abstract}

One of the most attractive materials for next generation electronics is graphene, because of its excellent electronic properties such as high carrier mobility ${ }^{1}$ and long electron coherent length. ${ }^{2}$ There have been, therefore, many studies on field effect transistors (FETs) using graphene layers as channels. $^{3-6}$ So far, several fabrication processes of a graphene channel (i.e., graphene nanoribbon) for FETs have been proposed. One is a top-down process that includes conventional electron beam lithography ${ }^{7}$ and scanning tunnel microscope lithography using anodic oxidation. ${ }^{8}$ Another is a bottom-up process such as graphite exfoliation by chemical methods ${ }^{9}$ and self-assembly technique using molecular precursors. ${ }^{10}$ Electron beam irradiation of graphene is naturally used in a conventional electron beam lithography. To fabricate the source, drain, and gate electrodes of graphene FET (GFET) structures, electron beam lithography is often adopted even if the graphene channel is fabricated by other means. In addition, scanning electron microscopy (SEM) is widely used for observations and tests of GFET structures. Thus, electron beam irradiation of graphene is unavoidable during GFET fabrication.

There are many reports on irradiation-induced damage in carbon materials, ${ }^{11}$ such as graphite, ${ }^{12-14}$ carbon nanotubes (CNTs), ${ }^{15-18}$ and graphene. ${ }^{19-21}$ Most of this literature has considered mainly knock-on damage (i.e., atomic displacement) caused by electron beams with energies higher than $86 \mathrm{keV} .^{16}$ Recently, however, several research groups have reported that damage occurs with low and medium electron energies in the range $1.0 \mathrm{keV}-50 \mathrm{keV}$ on $\mathrm{CNTs}^{22-24}$ and graphene. ${ }^{25}$ This energy range is commonly used in SEM and electron beam lithography. Therefore, electron beam processing would induce severe damage and defects to carbon-based devices, resulting in a deterioration of device performances. However, damage induced by low-energy irradiation in graphene has not been investigated in detail. It is further expected that damage induces internal strain. The damage and internal strain are quite important for practical

\footnotetext{
${ }^{\text {a) }}$ Author to whom correspondence should be addressed. Electronic mail: k.murakami@bk.tsukuba.ac.jp.
}

applications of GFET as well as for pure scientific interest. For the latter, mechanisms and lower limits of electron energies that induce such damage and strain have been unclear. For practical applications, the opening of the band gap in graphene by uniaxial strain has been theoretically reported. ${ }^{26}$ Therefore, strain induced by irradiation might provoke such an opening. From our study, we found that the damage to graphene was generated by very-low-energy irradiation of as little as $100 \mathrm{eV}$. Internal strain induced by such irradiation was quantitatively evaluated based on $G$ band peak shifts in the Raman spectra.

Single-layer graphene is deposited on $\mathrm{SiO}_{2}$ (thickness of $300 \mathrm{~nm}) / \mathrm{p}-\mathrm{Si}$ substrate by mechanical exfoliation of kish graphite using adhesive tape. Single-layer graphene is confirmed by Raman spectroscopy through $2 \mathrm{D}$ peak analysis. ${ }^{27}$ With the field emission SEM (Hitachi S-4800), an electron beam with energy range from $100 \mathrm{eV}$ (representing the lower limit of the equipment) to $10 \mathrm{keV}$ and various doses of up to $12 \mathrm{mC} / \mathrm{cm}^{2}$ was used to irradiate single-layer graphene at room temperature. A retarding potential is applied to the sample stage when electron energies were less than $500 \mathrm{eV}$ (e.g., accelerating and retarding voltages were $1.6 \mathrm{kV}$ and $1.5 \mathrm{kV}$, respectively, with a beam energy of $100 \mathrm{eV}$ ).

The series Raman spectroscopy maps for single-layer graphene before and after irradiation with various doses were measured for each accelerating voltage using the RAMANplus spectroscopy system (Nanophoton Corp.). The wavelength of incident laser light is $532 \mathrm{~nm}$. The laser power is reduced to $0.1 \mathrm{~mW}$ by a neutral density filter to avoid the change and shift of Raman peak by thermal effects induced by the incident laser light.

Figure 1 shows the series Raman spectra of single-layer graphene before and after $500 \mathrm{eV}$ irradiation with various doses. Before irradiation, graphene features a $\mathrm{G}$ peak, which is due to the doubly degenerate zone center $\mathrm{E}_{2 \mathrm{~g}}$ mode, ${ }^{28}$ and a large 2D peak, which corresponds to the second order of the $\mathrm{D}$ peak. ${ }^{27}$ The $\mathrm{D}$ peak appears after irradiation and is due to the breathing modes of the $\mathrm{sp}^{2}$ rings that require a defect for activation. ${ }^{28}$ The intensity of both $G$ and $2 D$ peaks decreases with increasing radiation dose. The $G$ peak width 


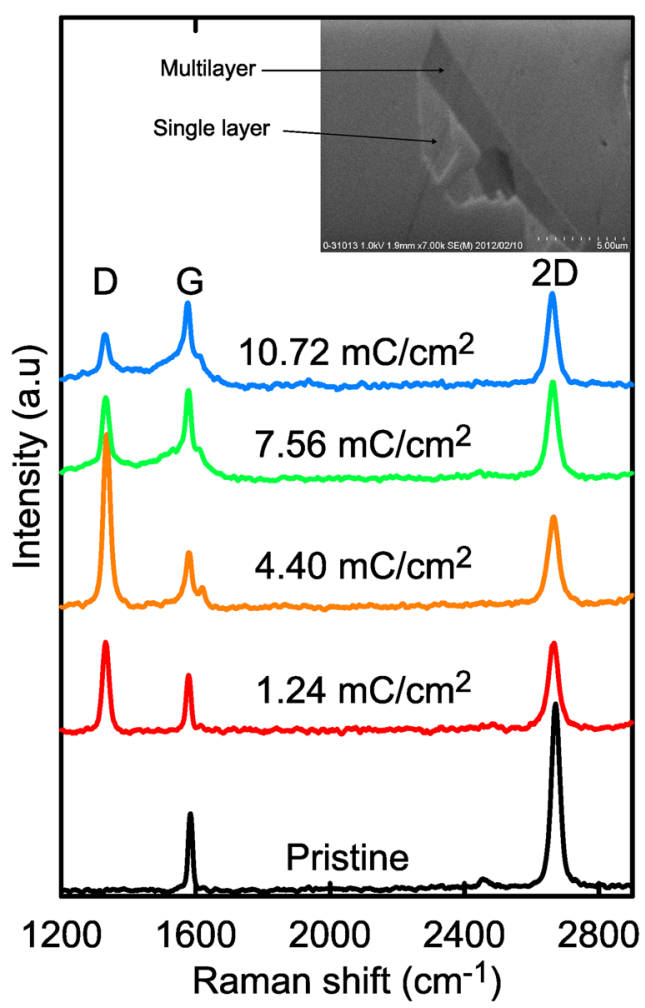

FIG. 1. Series Raman spectra of single-layer graphene before and after $500 \mathrm{eV}$ electron beam irradiation at various electron doses. Inset figure is the SEM image of a typical specimen of single-layer graphene.

becomes wider with higher dose; this tendency was observed under all irradiation conditions.

Figure 2 shows the intensity ratio for $D$ peak to $G$ peak (i.e., $\mathrm{I}(\mathrm{D}) / \mathrm{I}(\mathrm{G})$ ) and the $\mathrm{G}$ peak position before and after

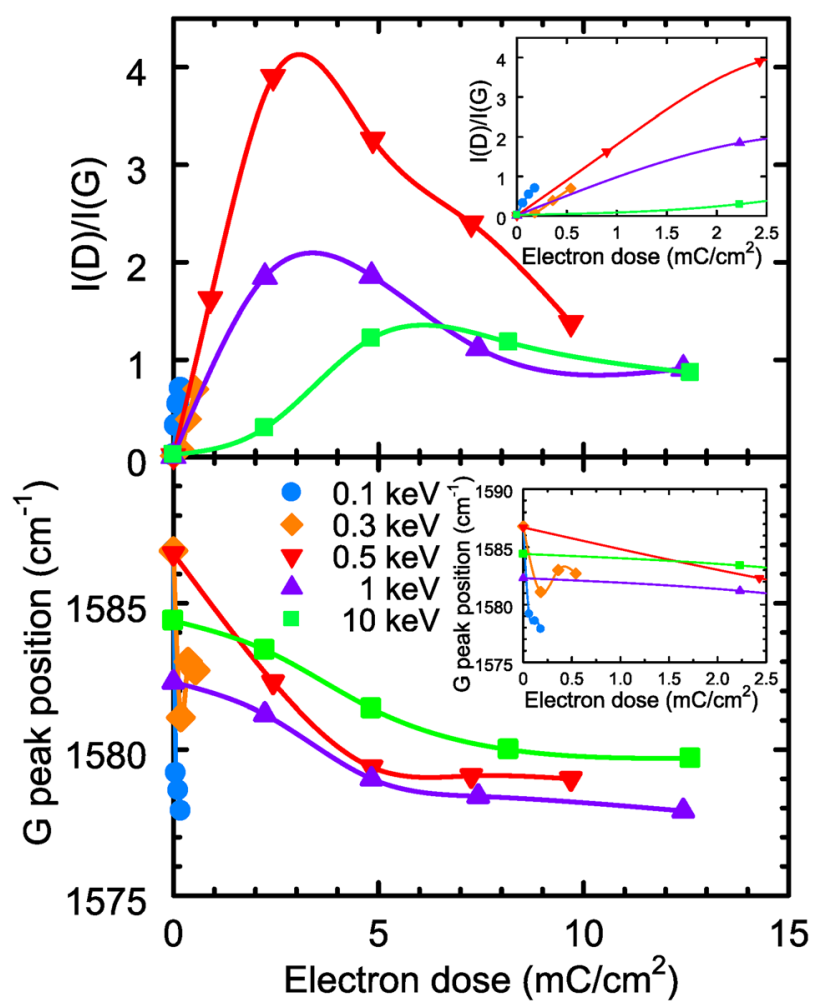

FIG. 2. The $I(D) / I(G)$ and $G$ peak position under irradiation with various electron energies as a function of electron dose. Inset figures are enlarged views in the low electron dose range $\left(0-2.5 \mathrm{mC} / \mathrm{cm}^{2}\right)$. irradiation with various electron energies as a function of electron dose. The $\mathrm{I}(\mathrm{D}) / \mathrm{I}(\mathrm{G})$ ratio increases with decreasing electron energy. Irradiation with energies as low as $100 \mathrm{eV}$ and low dose of around $0.25 \mathrm{mC} / \mathrm{cm}^{2}$ was found to induce damage in graphene. This result indicates that low-energy electrons can induce significant damage in graphene. Irradiation of energies $500 \mathrm{eV}, 1 \mathrm{keV}$, and $10 \mathrm{keV}$ first increases $\mathrm{I}(\mathrm{D}) / \mathrm{I}(\mathrm{G})$ ratio, reaching maximums, and then gradually decreases these ratios. This tendency is entirely consistent with an earlier report for $5 \mathrm{keV}$ - and $20 \mathrm{keV}$-irradiated graphene, ${ }^{25}$ and can be interpreted as the amorphization trajectory as proposed by Ferrari and Robertson. ${ }^{29}$ According to their interpretation for the $\mathrm{I}(\mathrm{D}) / \mathrm{I}(\mathrm{G})$ ratio of carbon materials, the ratio first increased when crystalline graphite becomes nanocrystalline graphite, and then decreased when nanocrystalline graphite is transformed into $\mathrm{sp}^{2}$ amorphous carbon. Under $500 \mathrm{eV}$ irradiation, crystalline graphene becomes nanocrystalline graphite under irradiation up to approximately $3 \mathrm{mC} / \mathrm{cm}^{2}$, and then, nanocrystalline graphite is further transformed into amorphous carbon due to higher irradiation damage. The $\mathrm{G}$ peak width broadens with dosages of greater than $4.40 \mathrm{mC} / \mathrm{cm}^{2}$ (Fig. 1), where the $\mathrm{I}(\mathrm{D}) / \mathrm{I}(\mathrm{G})$ ratios decrease from their maximum. This result also suggests that graphene undergoes amorphization under high irradiation dosages. The shift in $\mathrm{G}$ peak position, attributed to strains in graphene, is found to be larger with decreasing electron energy and saturates at higher electron dosages. The $\mathrm{G}$ peak position is red-shifted after irradiation, which indicates tensile straining in graphene. ${ }^{26}$ The defects generated by irradiation possibly induce internal strains in graphene. This strain can be quantitatively evaluated based on the $G$ peak shifts of graphene. To quantitatively evaluate strain, its type (i.e., uniaxial strain or biaxial strain) needs determining. 30

Figure 3 shows the Raman mapping of the $\mathrm{G}$ peak position after irradiation of $9.72 \mathrm{mC} / \mathrm{cm}^{2}$ at $500 \mathrm{eV}$. The mapping in red corresponds to wavenumber $1573.6 \mathrm{~cm}^{-1}$ where the $\mathrm{G}$ peak position shifts to lower wavenumber from the initial peak position of $1577.3 \mathrm{~cm}^{-1}$ (i.e., corresponding to the tensile strained regime). The mapping in blue corresponds to wavenumber $1581.1 \mathrm{~cm}^{-1}$ where the $\mathrm{G}$ peak position shifts to higher wavenumber from the initial peak position of $1577.3 \mathrm{~cm}^{-1}$ (i.e., corresponding to the compressive strained regime). Total strain in the entire graphene fragment seems to be compensated by tensile strain at the center of the graphene fragment and compressive strain at the surrounding edges of the graphene fragment. This result suggests that biaxial tensile strain (i.e., isotropic tensile strain) might be
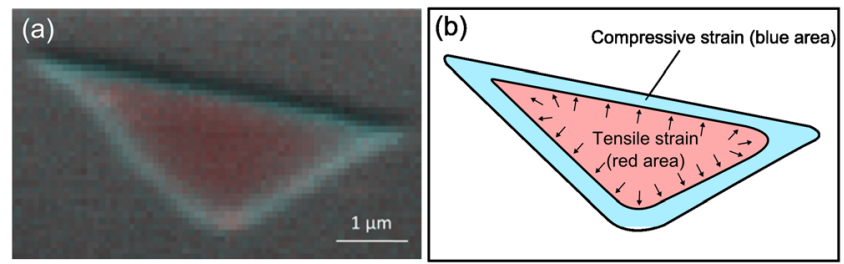

FIG. 3. (a) The mapping of $\mathrm{G}$ peak position after irradiation of $9.72 \mathrm{mC} / \mathrm{cm}^{2}$ at $500 \mathrm{eV}$. The mapping in red $\left(1573.6 \mathrm{~cm}^{-1}\right)$ and blue $\left(1581.1 \mathrm{~cm}^{-1}\right)$ corresponds, respectively, to the red-shifted $\mathrm{G}$ peak (i.e., tensile strain) and the blue-shifted $\mathrm{G}$ peak (i.e., compressive strain) from the initial $\mathrm{G}$ peak position before irradiation $\left(1577.3 \mathrm{~cm}^{-1}\right)$. (b) Schematic diagram of Fig. 3(a). 
induced by irradiation in the center of the graphene fragment. The $G$ peak shift dependence on biaxial tensile strain is expressed as ${ }^{30}$

$$
\Delta \omega_{G}=-2 \omega_{G}^{0} \gamma \varepsilon
$$

where $\Delta \omega_{G}$ is the $\mathrm{G}$ peak shift to the initial $\mathrm{G}$ peak position, $\omega_{G}^{0}$ the initial $\mathrm{G}$ peak position, $\gamma$ the Grüneisen parameter for doubly degenerate in-plane Raman-active $\mathrm{E}_{2 \mathrm{~g}}$ phonons, and $\varepsilon$ the biaxial strain. For this study, irradiation-induced internal strain is calculated by assuming biaxial strain and using $\gamma=1.8$, which is obtained from first-principles calculations. ${ }^{30}$ The calculated biaxial strain coefficient of $-57.0 \mathrm{~cm}^{-1} / \%$ is wholly consistent with that obtained experimentally from the graphene bubble. ${ }^{31}$

Figure 4 shows the biaxial tensile strain induced by irradiation with various energies as a function of dose. The dose-dependent internal strain in graphene shows obviously the same tendency as the dose-dependent $\mathrm{G}$ peak position for graphene. Namely, lower energy irradiation induces higher internal strain in graphene. These dose-dependent internal strains calculated from the linear part of the slopes (i.e., low electron dose range) were approximately $2.22 \% \mathrm{~cm}^{2} / \mathrm{mC}$ for $100 \mathrm{eV}$ and $2.65 \times 10^{-2} \% \mathrm{~cm}^{2} / \mathrm{mC}$ for $500 \mathrm{eV}$.

Here, we discuss the influence of finite carbon contamination during irradiation. It is well known that the reaction cross section of carbon contamination is higher for lower electron energies. ${ }^{32-34}$ In the case of lower-energy irradiation, the larger amount of carbon contaminants is deposited on graphene compared to the case of high-energy irradiation. It is very difficult to fully eliminate the large amount of carbon contamination deposited by low-energy irradiation in the experiment. The influence of carbon contamination is, therefore, an unavoidable issue in the low-energy irradiation experiment. There are several reports indicating that residual stress of deposited amorphous carbon films shows compressive strain. ${ }^{35-37}$ Carbon contamination should, therefore, induce compressive strain in graphene. However, in our experiment, lower-energy irradiation induces higher internal tensile strain in graphene. Consequently, tensile strain on graphene is not caused by the carbon contaminants. One of the most probable causes of damage and internal strain is bond breaking of graphene by low-energy irradiation. In the

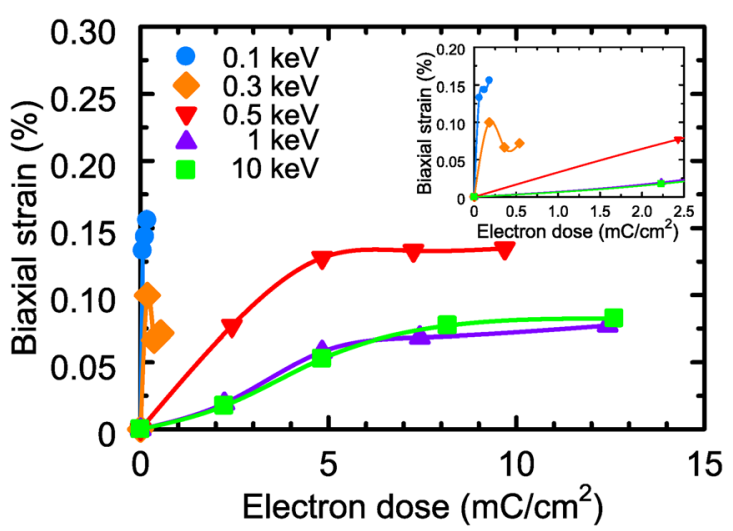

FIG. 4. The biaxial tensile strain induced by irradiation with various energies as a function of electron dose. Inset figure is the enlarged view in the low electron dose range $\left(0-2.5 \mathrm{mC} / \mathrm{cm}^{2}\right)$. case of low-energy irradiation (i.e., below knock-on threshold energy) for CNTs, Suzuki et al. reported that the irradiation damage occurs with bond breaking, which follows an electronic excitation induced by the energy of the incident electrons. ${ }^{38}$ In addition, the calculated electron inelastic mean free paths for CNTs become minimum around $100 \mathrm{eV}$, which means that low-energy irradiation around $100 \mathrm{eV}$ effectively excites the core-level and valence electrons, and plasmons due to the large strength of inelastic electron scattering. ${ }^{39}$ Another probable cause of damage and internal strain is the chemical etching of graphene by highly reactive $\mathrm{OH}$ radicals generated from water molecules on the surface under irradiation. ${ }^{23}$ The cross section for the ionization of a water molecule increases with decreasing electron energy and reaches to its maximum at around $100 \mathrm{eV} .^{40}$ The above two causes explain well why lower energy irradiation induces higher damage and strain on graphene in our experiments. Another source of internal strain occurs in the transition during irradiation from an $\mathrm{sp}^{2}$-bonded honey-comb lattice to an $\mathrm{sp}^{3}$-bonded 3D network. ${ }^{13,41}$

The maximum internal tensile strain calculated in this study is approximately $0.16 \%$ at $100 \mathrm{eV}$ irradiation with dose of $0.18 \mathrm{mC} / \mathrm{cm}^{2}$, which is insufficient for band gap opening, because a $0.8 \%$ strain is theoretically required for the $300 \mathrm{meV}$ band gap opening. ${ }^{26}$ In addition, a uniaxial tensile strain in graphene is required to control its band gap. Therefore, the controlling of the strain direction and larger induced strain are necessary if irradiation is to open a band gap in graphene. One possible method to control strain direction is patterning the irradiation in graphene. For example, a periodic line pattern would induce uniaxial tensile strain perpendicular to the line direction over the irradiated area. Further study on this effect is being undertaken.

We found that a very low-energy electron beam can strongly induce damage in graphene, even if electron energies are only $100 \mathrm{eV}$. Lower-energy electrons induced larger damage at smaller doses. The biaxial tensile strain at the center of the graphene fragment was found to be induced by irradiation from the mapping of the $\mathrm{G}$ peak shift. The internal biaxial tensile strain was quantitatively evaluated based on $G$ peak shifts. Lower-energy electrons induced larger strain. Such additionally induced damage and strain should serve as a barrier that decreases ballistic electron transport. Alternatively, artificially induced damage under controlled electron dosage might provide a new method to fabricate electron confinement structures on graphene such as 1D graphene nanoribbons.

This work was partly supported by Ministry of Education, Culture, Sports, Science and Technology (MEXT) of Japan, through a Grant-in-Aid for Scientific Research A (23246063) and Nanotechnology Network Project.

${ }^{1}$ K. S. Novoselov, A. K. Geim, S. V. Morozov, D. Jiang, Y. Zhang, S. V. Dubonos, I. V. Grigorieva, and A. A. Firsov, Science 306, 666 (2004).

${ }^{2}$ C. Berger, Z. Song, X. Li, X. Wu, N. Brown, C. Naud, D. Mayou, T. Li, J. Hass, A. N. Marchenkov, E. H. Conrad, P. N. First, and W. A. de Heer, Science 312, 1191 (2006).

${ }^{3}$ C. Berger, Z. Song. T. Li, X. Li, A. Y. Ogbazghi, R. Feng, Z. Dai, A. N. Marchenkov, E. H. Conrad, P. N. First, and W. A. de Heer, J. Phys. Chem. B 108, 19912 (2004). 
${ }^{4}$ Q. Yan, B. Huang, J. Yu, F. Zheng, J. Zahng, J. Wu, B.-L. Gu, F. Liu, and W. Duan, Nano Lett. 7, 1469 (2007).

${ }^{5}$ B. Özyilmaz, P. Jarillo-Herrero, D. Efetov, and P. Kim, Appl. Phys. Lett. 91, 192107 (2007).

${ }^{6}$ Y.-M. Lin, A. Valdes-Garcia, S.-J. Han, D. B. Farmer, I. Meric, Y. Sun, Y. Wu, C. Dimitrakopoulos, A. Grill, P. Avouris, and K. A. Jenkins, Science 332, 1294 (2011).

${ }^{7}$ M. Y. Han, B. Özyilmaz, Y. Zhang, and P. Kim, Phys. Rev. Lett. 98, 206805 (2007).

${ }^{8}$ L. Tapasztó, G. Dobrik, P. Lambin, and L. P. Brió, Nat. Nanotechnol. 3, 397 (2008).

${ }^{9}$ X. Li, X. Wang. L. Zhang, S. Lee, and H. Dai, Science 319, 1229 (2008).

${ }^{10}$ J. Cai, P. Ruffieux, R. Jaafar, M. Bieri, T. Braun, S. Blankenburg, M. Muoth, A. P. Seisonen, M. Saleh, X. Feng, K. Müllen, and R. Fasel, Nature 466, 470 (2010).

${ }^{11}$ F. Banhart, Rep. Prog. Phys. 62, 1181 (1999).

${ }^{12}$ J. Koike and D. F. Pedraza, J. Mater. Res. 9, 1899 (1994).

${ }^{13}$ M. Takeuchi, S. Muto, T. Tanabe, S. Arai, and T. Kuroyanagi, Philos. Mag. A 76, 691 (1997).

${ }^{14}$ R. H. Telling and M. I. Heggie, Philos. Mag. 87, 4797 (2007).

${ }^{15}$ F. Beuneu, C. 1'Huillier, J.-P. Salvetat, J.-M. Bonard, and L. Forró, Phys. Rev. B 59, 5945 (1999).

${ }^{16}$ B. W. Smith and D. E. Luzzi, J. Appl. Phys. 90, 3509 (2001).

${ }^{17}$ F. Banhart, J. X. Li, and A. V. Krasheninnikov, Phys. Rev. B 71, 241408 (2005).

${ }^{18}$ K. Mølhave, S. B. Gudnason, A. T. Pedersen, C. H. Clausen, A. Horsewell, and P. Bøggild, Ultramicrosocpy 108, 52 (2007).

${ }^{19}$ A. Hashimoto, K. Suenaga, A. Gloter, K. Urita, and S. Iijima, Nature 430, 870 (2004).

${ }^{20}$ J. C. Meyer, C. Kisielowski, R. Erni, M. D. Rossell, M. F. Crommie, and A. Zettl, Nano Lett. 8, 3582 (2008).

${ }^{21}$ J. C. Meyer, F. Eder, S. Kurasch, V. Skakalova, J. Kotakoski, H. J. Park, S. Roth, A. Chuvilin, S. Eyhusen, G. Benner, A. V. Krasheninnikov, and U. Kaiser, Phys. Rev. Lett. 108, 196102 (2012).

${ }^{22}$ S. Suzuki, K. Kanzaki, Y. Homma, and S. Fukuba, Jpn. J. Appl. Phys., Part 2 43, L1118 (2004).
${ }^{23}$ T. D. Yuzvinsky, A. M. Fennimore, W. Micelson, C. Esquivias, and A. Zettl, Appl. Phys. Lett. 86, 053109 (2005).

${ }^{24}$ S. Gupta and R. J. Patel, J. Raman Spectrosc. 38, 188 (2006).

${ }^{25}$ D. Teweldebrhan and A. A. Balandin, Appl. Phys. Lett. 94, 013101 (2009).

${ }^{26}$ Z. H. Ni, T. Yu, T. H. Lu, Y. Y. Wang, Y. P. Feng, and Z. X. Shen, ACS Nano 2, 2301 (2008).

${ }^{27}$ A. C. Ferrari, J. C. Meyer, V. Scardaci, C. Casiraghi, M. Lazzeri, F. Mauri, S. Piscanec, D. Jiang, K. S. Novoselov, S. Roth, and A. K. Geim, Phys. Rev. Lett. 97, 187401 (2006).

${ }^{28}$ F. Tuinstra and J. L. Koenig, J. Chem. Phys. 53, 1126 (1970).

${ }^{29}$ A. C. Ferrari and J. Robertson, Phys. Rev. B 61, 14095 (2000).

${ }^{30}$ T. M. G. Mohiuddin, A. Lombardo, R. R. Nair, A. Bonetti, G. Savini, R. Jalil, N. Bonini, D. M. Basko, C. Galiotis, N. Marzari, K. S. Novoselov, A. K. Geim, and A. C. Ferrari, Phys. Rev. B 79, 205433 (2009).

${ }^{31}$ J. Zabel, R. R. Nair, A. Ott, T. Georgiou, A. K. Geim, K. S. Novoselov, and C. Casiraghi, Nano Lett. 12, 617 (2012).

${ }^{32}$ K. I. Schiffmann, Nanotechnology 4, 163 (1993).

${ }^{33}$ H. W. P. Koops, A. Kaya, and M. Weber, J. Vac. Sci. Technol. B 13, 2400 (1995).

${ }^{34}$ A. Botman, D. A. M. de Winter, and J. J. L. Mulders, J. Vac. Sci. Technol. B 26, 2460 (2008).

${ }^{35}$ J. P. Sullivan, T. A. Friedmann, and A. G. Baca, J. Electron. Mater. 26, 1021 (1997).

${ }^{36}$ C. Charitidis, S. Logothetidis, and P. Douka, Diamond Relat. Mater. 8, 558 (1999).

${ }^{37}$ S. E. Ong, S. Zhang, H. Du, and D. Sun, Diamond Relat. Mater. 16, 1628 (2007).

${ }^{38}$ S. Suzuki, K. Yamaya, Y. Homma, and Y. Kobayashi, Carbon 48, 3211 (2011).

${ }^{39}$ I. Kyriakou, D. Emfietzoglou, R. G-Molina, I. Abril, and K. Kostarelos, Appl. Phys. Lett. 94, 263113 (2009).

${ }^{40}$ J. Schutten, F. J. de Heer, H. R. Moustafa, A. J. H. Boerboom, and J. Kistemaker, J. Chem. Phys. 44, 3924 (1966).

${ }^{41}$ T. Tanabe, Phys. Scr. T64, 7 (1996). 